\title{
Levantamento dos casos de Leishmaniose Visceral (calazar) em cães e humanos no município de Guaraí, Tocantins entre 2015-2020
}

Survey of Visceral Leishmaniasis (calazar) cases in dogs and human in the municipality of Guaraí, Tocantins between 2015-2020

Encuesta de casos de Leishmaniasis Visceral (calazar) en perros y humanos en el municipio de Guaraí, Tocantins entre 2015-2020

Recebido: 28/11/2021 | Revisado: 03/12/2021 | Aceito: 04/12/2021 | Publicado: 04/12/2021

Karolayne Bernardes Dos Santos ORCID: https://orcid.org/0000-0002-2706-9302 Faculdade Guaraí, Brasil E-mail: karol.karolayne@ hotmail.com

Mirian Barreira Martins ORCID: https://orcid.org/0000-0001-7850-6296 Faculdade Guaraí, Brasil

E-mail: miahcorcino@gmail.com

Aluísio Vasconcelos de Carvalho ORCID: https://orcid.org/0000-0002-3793-3133 Faculdade Guaraí, Brasil

E-mail: aluisiovasconcelos@gmail.com

\begin{abstract}
Resumo
A Leishmaniose Visceral, conhecida como calazar no Brasil, é causada pelo agente etiológico Leishmania infantum chagasi a transmissão da doença ocorre por intermédio da picada da fêmea de um invertebrado hematófago pertencente à família dos flebótomos, popularmente conhecido como mosquito-palha, doença que causa sintomas clínicos podendo afetar tanto animais como humanos. O presente trabalho objetiva-se em averiguar o percentual de casos de infecções da doença no município de Guaraí, Tocantins, em humanos e animais (cães), buscando compreender os fatores que influenciam a prevalência da doença no município. Trata-se de uma pesquisa documental, com abordagem quantitativa. Os dados foram obtidos diante a análises de documentos contidos na Secretaria municipal de Saúde e no Centro de Controle de Zoonoses do município entre os anos de 2015 a 2020 . Através do presente estudo, percebeu-se que a taxa de afetados pela doença em seres humanos chega a ser bem menor que em animais, pois dentro do período analisado foram totalizados 15 testes confirmados com a doença em seres humanos e 1.752 testes confirmados em animais. Devem ser adotadas políticas públicas que minimizem os efeitos gerados pela doença tanto em animais e humanos, redução da quantidade de lixo, reduzir cães errantes e adoção de educação ambiental no município.
\end{abstract}

Palavras-chave: Leishmaniose visceral; Humanos; Animais; Doenças; Causas.

\begin{abstract}
Visceral Leishmaniasis, known as calazar in Brazil, is caused by the etiological agent Leishmania infantum chagasi transmission of the disease occurs through the bite of the female of a hematophagous invertebrate belonging to the family of phlebotomies, popularly known as straw mosquito, a disease that causes clinical symptoms that can affect both animals and humans. The present study aims to investigate the percentage of cases of disease infections in the municipality of Guaraí, Tocantins, in humans and animals (dogs), seeking to understand the factors that influence the prevalence of the disease in the municipality. This is documentary research, with a quantitative approach. The data were obtained from the analysis of documents contained in the municipal Health Department and the Zoonosis Control Center of the municipality between the years 2015 to 2020 . Through the present study, it was noticed that the rate of affected by the disease in humans is much lower than in animals, because within the analyzed period, 15 tests confirmed with the disease were totaled in humans and 1,752 confirmed tests in animals. Public policies should be adopted to minimize the effects generated by the disease in both animals and humans, reducing the amount of litter, reducing errant dogs and adopting environmental education in the municipality.
\end{abstract}

Keywords: Visceral Leishmaniasis; Humans; Animals; Diseases; Causes.

\section{Resumen}

La leishmaniasis visceral, conocida como calazar en Brasil, es causada por el agente etiológico Leishmania infantum chagasi La transmisión de la enfermedad ocurre a través de la picadura de la hembra de un invertebrado hematófago 
perteneciente a la familia de los flebotomes, popularmente conocido como mosquito de paja, una enfermedad que causa síntomas clínicos que pueden afectar tanto a animales como a humanos. El presente estudio tiene como objetivo investigar el porcentaje de casos de infecciones por enfermedades en el municipio de Guaraí, Tocantins, en humanos y animales (perros), buscando comprender los factores que influyen en la prevalencia de la enfermedad en el municipio. Se trata de una investigación documental, con un enfoque cuantitativo. Los datos se obtuvieron del análisis de documentos contenidos en el Departamento de Salud municipal y el Centro de Control de Zoonosis del municipio entre los años 2015 a 2020. A través del presente estudio, se observó que la tasa de afectados por la enfermedad en humanos es mucho menor que en animales, pues dentro del periodo analizado se totalizaron 15 pruebas confirmadas con la enfermedad en humanos y 1.752 pruebas confirmadas en animales. Se deben adoptar políticas públicas para minimizar los efectos generados por la enfermedad tanto en animales como en humanos, reduciendo la cantidad de basura, reduciendo los perros errantes y adoptando la educación ambiental en el municipio.

Palabras clave: Leishmaniasis visceral; Humanos; Animales; Enfermidades; Causas.

\section{Introdução}

A Leishmaniose constitui um conjunto de doenças ocasionadas por protozoários do gênero Leishmania, família Trypanosomatidae que acometem em seres humanos e outros mamíferos. A transmissão da doença ocorre por intermédio da picada da fêmea de um invertebrado hematófago pertencente à família dos flebótomos, popularmente conhecido como mosquito-palha (Azevedo et al., 2021).

A doença possui dois tipos, a Leishmaniose Tegumentar (LT), no qual afeta a pele e membranas mucosas, e Leishmaniose visceral (LV) em que acomete os órgãos internos. A LV, também chamada de Calazar, é uma doença que ocorre em diversos países do mundo sendo responsável por cerca de 59.000 mil óbitos todos os anos. A presença endêmica da Leishmaniose visceral está distribuída em 98 países com aumento progressivo no número de ocorrência, a Organização Mundial da Saúde em 2015 relatou que mais de 90\% dos casos identificados ocorreram em cinco países do globo: Brasil, Índia, Sudão, Sudão do Sul e Bangladesh (Who, 2015).

No território brasileiro, até a década de 70, a Leishmaniose Visceral era considerada uma doença com transmissão doméstica e pet-doméstica de ocorrência essencialmente rural. Contudo, nas últimas décadas houve uma expansão do campo geográfico e da magnitude de transmissão da doença elevando o problema para os centros urbanos, inclusive para as maiores metrópoles do país. A expansão da dimensão da doença, em relação a progressão para os centros urbanos é devido a alguns fatores, entre eles destacam-se aumento da densidade populacional nos centros urbanos, intensas migrações ocorrentes nas últimas décadas, inadequação das condições de vida de determinada parte da população, alterações ambientais e adaptação do vetor ao meio urbano (Almeida et al., 2020).

O Estado do Tocantins é um dos lugares do Brasil onde há uma das maiores taxas de incidência de Leishmaniose Visceral, constituindo, entre o período de 2007 a 2014, um número de 26,2 infectados/100 mil habitantes. Estudos de análises de vulnerabilidade de transmissão de Leishmaniose Visceral Humana (LVH) por análise de distribuição espacial de LV constatou que algumas variáveis contribuem diretamente para a propagação da doença, entre essas variáveis destacam-se: modelo suburbano restrito, ou seja, alta intensidade de surtos da doença em regiões periféricas; padrão periurbano difuso, referindo-se a um elevado número de casos localizados em regiões urbanas periféricas, com pouca incidência nas áreas centrais de municípios; epidemiologia de disseminação centrífuga, ao que corresponde a disseminação da doença no ambiente municipal estendendo-se a municípios vizinhos e por último foi identificado padrão de disseminação em rede, em diversos municípios entram em surtos sequenciais da doenças (Reis et al., 2019).

Entre os fatores internos que potencializam a propagação de doenças infecciosas em uma localidade específica, Teixeira (2019), afirma que a distribuição desigual de renda, problemas socioeconômicos, falta de educação e falta de saneamento básico são os fatores de ter um impacto maior na disseminação dessas doenças. Nessa percepção, o entendimento do processo de vida e dos meios de transmissão da Leishmaniose visceral é de grande importância para a profilaxia da doença, visto que as áreas onde há uma grande densidade de pessoas com baixa escolaridade e qualidade de vida são as regiões com 
maiores índices de contaminação e propagação da doença.

O presente trabalho objetiva-se em averiguar o percentual de casos de infecções da doença no município de Guaraí, Tocantins, em humanos e animais (cães), buscando compreender os fatores que influenciam a prevalência da doença no município.

\section{Metodologia}

\section{1 Área de estudo}

O estudo foi realizado no município de Guaraí pertencente ao Estado do Tocantins (Figura 1), situado a 178 quilômetros da capital Palmas. A cidade possui uma área total de $2.275 .562 \mathrm{~km}^{2}$ e está localizada a uma latitude $08^{\circ} 50^{\prime} 03^{\prime \prime}$ sul e a uma longitude $48^{\circ} 30^{\prime} 37^{\prime \prime}$ oeste, se encontrando a uma altitude de 259 metros.

Figura1. Localização geográfica do município de Guaraí - TO.

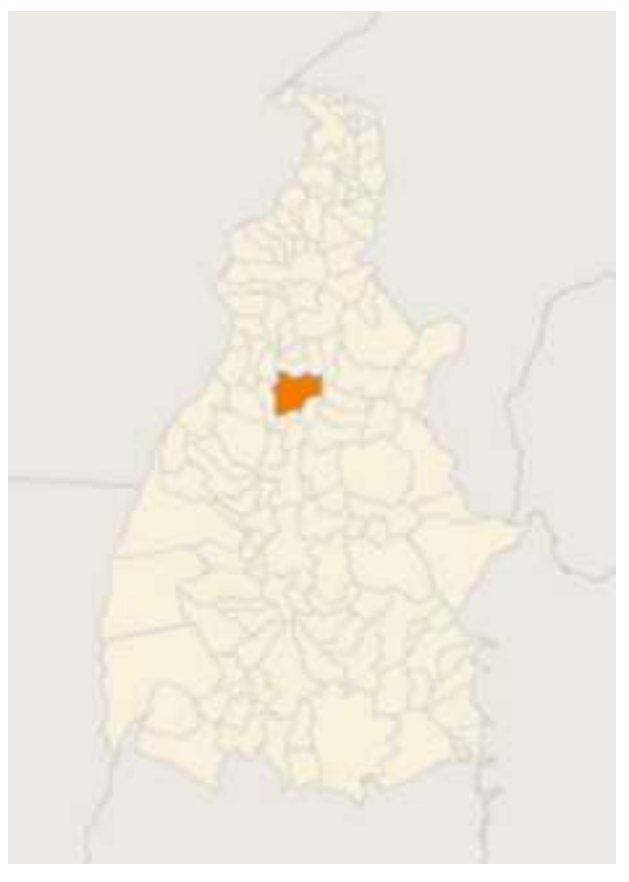

Fonte: IBGE (2010).

\subsection{Percurso Metodológico}

Trata-se de uma pesquisa documental, com abordagem quantitativa. Utilizou-se para a pesquisa o método estatístico que consiste em quantificar dados sobre acontecimentos, fatos, fenômenos, visando analisar as relações que diversos fatos e fenômenos estabelecem entre si (Mazucato, 2018). A coleta de dados foi feita por meio de documentos contidos na Secretaria Municipal de Saúde e no Centro de Controle De Zoonoses do município. Inicialmente foi coletado informações acerca dos índices de casos de Leishmaniose Visceral referente a cada ano entre 2015 a 2020. Após o levantamento das informações, utilizou-se estatística descritiva para análise dos dados afim de compreender suas causalidades.

\section{Resultados e Discussão}

Observou-se que houve variação nos dados apresentados em cães e humanos, isso também provou a estreita relação entre os casos de ambos, além das condições ambientais adversas que influencia as notificações. 
De acordo com as observações, 24 casos foram notificados em 2015 na cidade ( 3 confirmados), já em 2016 houve uma redução nos casos, apenas 13 testes realizados na cidade ( 3 positivos), no entanto, em 2017 ocorreram 22 testes (5 positivos), em 2018, 17 testes (1 positivo), em 2019 foram realizados 15 testes (3 positivos), e por fim, em 2020 foram registrados 9 testes realizados na cidade e nenhum caso foi detectado como positivo (Figura 2).

Figura 2. Leishmaniose visceral humana.

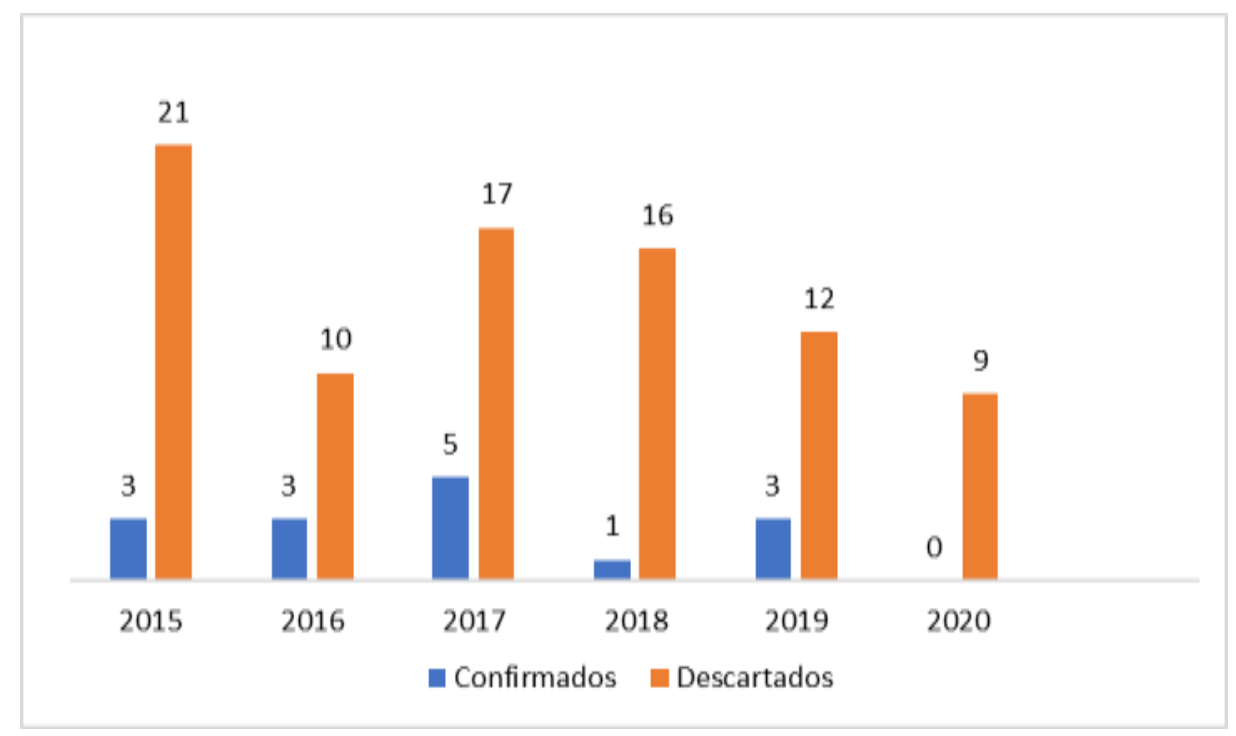

Fonte: SINAN/ SEMUSA Guaraí /Gerência de Sistemas.

No Brasil o primeiro caso de Leishmaniose Visceral relatado ocorreu no ano de 1934 pelo patologista Henrique Penna da fundação Rockfeller, a descoberta ocorreu através da análise de cortes histológico de fragmentos de fígados de pessoas que supostamente teriam falecido de febre amarela, entretanto, o patologista encontrou formas amastigotas protozoário do gênero Leishmania em 41 das amostras averiguadas, principalmente em crianças provenientes das regiões nordeste e norte do país. Com a divulgação dos achados de Penna foi confirmado a autóctone com elevada ocorrência da doença nas Américas (Benchimol et al., 2019).

A Leishmaniose Visceral é culpada por cerca de 59.000 óbitos por ano ao redor do mundo e quantifica-se que cerca 12 milhões de pessoas são infectadas todos os anos. No Brasil, averiguando os dez anos últimos anos, foram identificados 42.067 casos, no qual destes ocorreram 2.704 mortes, com a média de incidência de 1,92/100.000 habitantes. Desse modo, a LV é uma doença que atinge todas as faixas etárias da população, sem agravamento por gênero ou raça, contudo, a maior parte da população acometida é a população residentes em áreas com baixo índice de saneamento básico e poucas condições socioeconômicas (Brasil, 2014).

No Brasil, existem parâmetros para catalogação de locais para a vigilância e manejo da LV nos municípios, que visa compreender qualitativamente o risco e a intensidade da transmissão da doença. Visando as práticas de vigilância e controle, e a fim de preferir o arrendamento de recursos humanos e financeiros, os municípios endêmicos foram estratificados segundo a intensidade de transmissão, e como critério, empregou-se a média anual de casos novos autóctones dos últimos três anos, segundo o município de infecção, conforme os dados inscritos no sistema de informação de agravos de notificação (SINAN). Os municípios são classificados, desta forma em transmissão esporádica ( $<2,4$ casos), transmissão moderada ( $\geq 2,4$ e $<4,4$ casos), transmissão intensa ( $\geq 4,4$ casos) e sem transmissão. Quando o número de casos humanos é acima do esperado em municípios com transmissão, existe uma situação de surto (Brasil, 2017).

A Leishmania chagasi é um protozoário tripanosomatídeo, com uma forma flagelada ou promastigota, encontrada no tubo digestivo do inseto vetor, e outra aflagelada ou amastigota, encontrada nos tecidos dos vertebrados (Gonçalves et al., 
1986; Rey, 1991; Castro et al., 1996). A transmissão se dá entre os animais e o homem pela picada da fêmea do inseto vetor, um díptero pertencente à subfamília Phlebotominae, conhecido comumente como flebótomo, da espécie Lutzomyia longipalpis (Castro et al., 1996).

Em 1936, Deane e Mangabeira responsabilizam esta espécie vetora por ser aquela mais frequentemente encontrada nas casas próximos às matas e por ter sido encontrada infectada após sugar um cão doente (Alencar, 1958; Leite, 1958; Oliveira, 1960; Pessoa, 1967; Gonçalves et al., 1986; Killick-Kendrick, 1990).

Sendo este vetor bem adaptável ao peridomicílio doméstico, o seu controle é muito importante na interrupção da doença. O conhecimento de sua dinâmica é essencial para o adequado controle de sua população (Morrison et al., 1995; Ferro et al., 1995; López et al., 1996).

Após ter sido verificada a incidência da doença geral diminuiu na década de 1950, mas o número de registros aumentou gradualmente nos últimos 20 anos. Atualmente, a Leishmaniose visceral atinge 17 estados brasileiros, principalmente do Nordeste, responsável por $94 \%$ dos casos registrados nesta década, e é observada a sua presença em centros urbanos, sob a forma de epidemias (Genaro et al., 1989; Costa et al., 1990; Tesh, 1995 e Castro et al., 1996).

Quanto aos casos de Leishmaniose visceral animal no município de Guaraí do Tocantins, os dados apresentados foram levantados pelo Centro de Controle de Zoonoses de Guaraí - TO. Pode-se observar que em 2015, foram examinados 712 casos na cidade (237 positivos), em 2016, ocorreu uma queda de 17\% nos casos examinados, porém houve aumento nos casos confirmados: foram 591 exames (247 positivos), em 2017 foram examinados 730 animais (310 positivos), em 2018 houve a maior alta dos anos, mais de 58\% de examinados com suspeita da doença na cidade, 1055 casos examinados (364 positivos), em 2019 foram realizados 868 exames (352 positivos), por fim, em 2020 foram 631 exames (239 positivos), conforme observado na Figura 3.

Figura 3. Leishmaniose visceral animal.

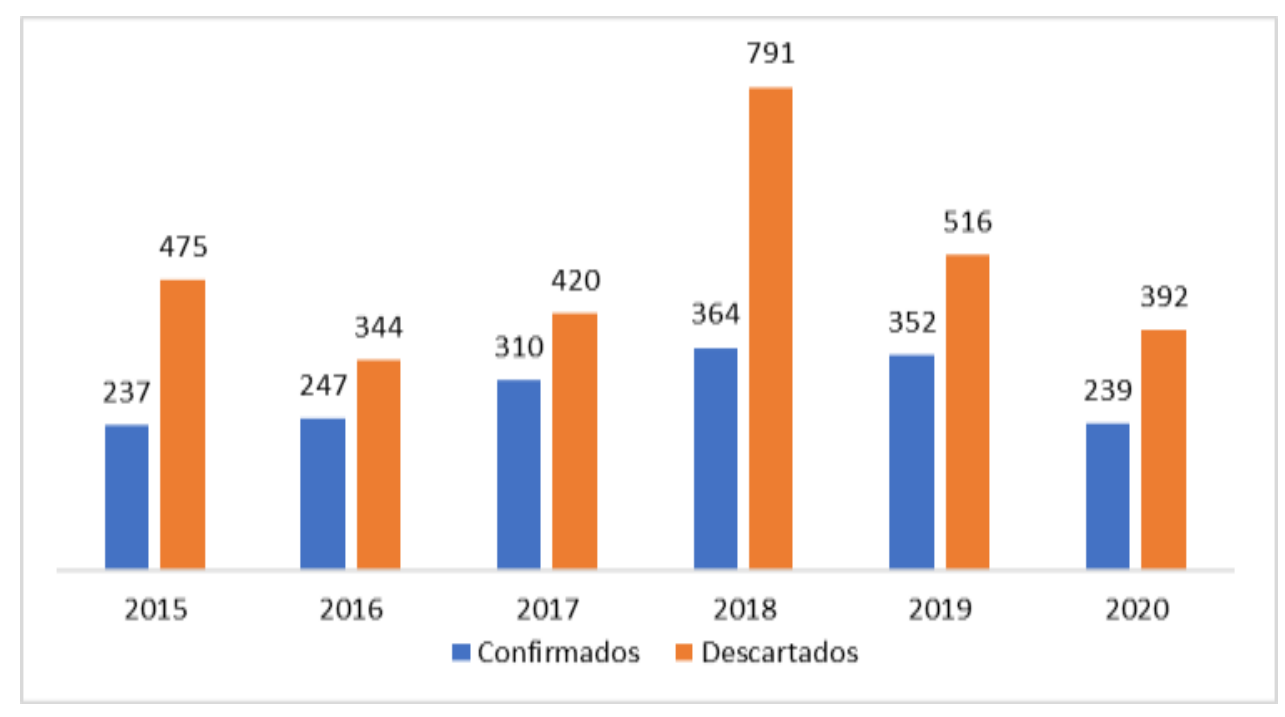

Fonte: CCZ-Guaraí.

No ano de 1936, Evandro Chagas realizou a primeira descrição clínica de um paciente vivo de LV no Brasil, e concluiu que a mesma diferia morfologicamente da Leishmaniose donovani agente causador do calazar em partes da Ásia, África e na Índia. Nos estudos realizados por Evandro Chagas foi identificado também diversas espécies de animais de estimação contaminados pelo protozoário Leishmaniose infantum, além de flebotomíneos da espécie Lutzomyia longipalpis (Benchimol et al., 2019). 
Atualmente a Organização Mundial da Saúde (OMS) considera a Leishmaniose visceral como um Doença Tropical Negligenciada (DTN) no qual expõe mais de 350 milhões de pessoas ao perigo de contaminação (Who, 2021). Nesse sentido, segundo a Organização Pan-Americana de Saúde, a LV é definida como endêmica em 12 países nos continentes americanos, sendo que o Brasil concentra cerca de $96 \%$ das ocorrências dos casos, com 7,9\% de taxa de letalidade, principalmente nas regiões Nordeste, Sudeste e Centro-Oeste e Norte do país (Opas, 2018).

Segundo Montalvo et al., (2012), 90\% dos casos registrados de LV ocorrem em países como a Índia, Bangladesh, Nepal, Sudão e Brasil, onde grande parte da população vive em situação de pobreza. No Brasil foram registrados 42.067 doentes nos últimos 12 anos, com ocorrência de 2.704 óbitos, resultando em uma incidência média de 1,92 casos por 100.000 habitantes durante este período (Bastos et al., 2015).

Nas áreas urbanas, os cães (Canis familiaris) são a principal fonte de infecção. A enzootia canina tem seguimento no incidente de casos humanos e a infecção em cães tem sido mais comum do que no homem. No ambiente silvestre, os reservatórios são as raposas (Dusicyon vetulus e Cerdocyon thous) e os marsupiais (Didelphis albiventris).

No Brasil, foram encontradas nas regiões Nordeste, Sudeste e na Amazônia raposas infectadas. No Brasil e na Colômbia foram encontrados infectados marsupiais didelfídeos. Até o momento, a infecção do animal não tem tendências raciais, de gênero ou relacionadas à idade. Em cães suscetíveis após infecção de pele, os parasitas se espalham por todo o corpo conforme os sintomas se desenvolvem. Dependendo das características do parasita e do hospedeiro, a leishmaniose canina pode se desenvolver de forma aguda ou crônica.

Entre vários motivos, a resposta dos linfócitos T tem o maior impacto na infecção. Como a Leishmania é um parasita intracelular obrigatório, a defesa do hospedeiro depende da atividade dessas células, que são reduzidas durante a infecção. Por outro lado, os linfócitos B proliferam em grandes quantidades e produzem grande quantidade de anticorpos, mas são prejudiciais e não têm efeito protetor. Portanto, os sintomas dependem da presença da imunocompetência do animal. Normalmente, a doença no cão é crônica, mas a evolução aguda e grave pode levar o animal ao óbito em pouco tempo. Em alguns cães a doença pode manter-se oculta, levando inclusive à cura espontânea. No Brasil, é encontrada com índices variados a forma assintomática da doença, em geral representa 40 a $60 \%$ de uma população soropositiva (Brasil, 2006).

De acordo com Marcondes e Rossi (2014), ao longo da década de 1990 no Brasil, devido ao processo de urbanização, a distribuição geográfica da doença se expandiu muito, levando a importantes epidemias em várias cidades da região Nordeste - São Luís, Natal e Aracaju, Norte Boa Vista e Santarém, Sudeste-Belo Horizonte e Montes Claros e Centro-Oeste-Cuiabá e Campo Grande.

A Organização Mundial de Saúde (OMS), como ferramentas de controle, preconiza três medidas principais, como: destruição do inseto vetor, tratamento dos casos humanos e eliminação dos reservatórios (cães sintomáticos e soropositivos) (Malaquias et al., 2007).

Segundo Bueno (2001) a educação para a saúde e a participação da comunidade são consideradas as formas mais importantes de combate às doenças e a cooperação pública tornou-se a base para o sucesso da campanha LVH. Seja através dos meios de comunicação de massa como televisão, rádio ou jornal, seja através de profissionais de saúde, por meio de visitas domiciliares ou palestras, as pessoas devem obter informações sobre doenças, vetores, medidas preventivas e de controle para que saibam como agir para ajudar a reduzir e posteriormente eliminar.

O diagnóstico da LVC ainda é uma adversidade, por não existir um teste que seja 100\% sensível e específico, e a comunidade de clínicos veterinários tem dificuldade na padronização dos testes sorológicos aplicados nos consultórios por serem unicamente qualitativos (Pinto et al., 2016). Os métodos de diagnóstico para LVC atualmente utilizados são a avaliação 
clínica, testes bioquímicos, parasitológicos, sorológicos, imunológicos e moleculares, podendo estar associados ou não (Dotta et al., 2009).

Não existem sinais e sintomas específicos da LVC devido a gama de manifestações que podem ser confundidas com outras patologias (Lima et al., 2013), entretanto alguns sinais clínicos são mais frequentes nos caninos como a apatia, onicogrifose, caquexia, ceratoconjutivite, linfadenopatia, hepatoesplenomegalia, úlceras de pele, febre, alopecia, descamação da pele, eczema, úlceras de mucosa, vômitos, sangramento retal e formação de edemas. Os sinais clínicos quando associados aos exames hematológicos e bioquímicos corroboram para o diagnóstico e tratamento da LVC (Freitas et al., 2012).

De acordo com os sinais clínicos externos da LVC, os cães infectados podem ser classificados em: caninos assintomáticos com nenhum sinal ou sintoma; caninos oligossintomáticos com até três sinais clínicos, entre eles: adenopatia linfóide, perda de peso e pelo opaco; e caninos sintomáticos com mais de três sinais clínicos principalmente alterações cutâneas (alopecia, descamação, úlceras e hiperqueratose), onicogrifose, emagrecimento e ceratoconjuntivite (Godoy et al., 2016). O diagnóstico clínico por meio de anamnese do animal pode ser um ponto limitante em relação aos sintomas semelhantes a outras enfermidades, necessitando complementação por meio de exames laboratoriais (Lima et al., 2013).

\section{Conclusão}

Através de dados obtidos na Secretaria da Saúde de Guaraí e no Centro de Controle de Zoonoses foi observado que a Leishmaniose é uma doença prevalente em ambas as espécies, o que caracteriza o espaço urbano um ambiente favorável para a manutenção dos flebótomos e seus reservatórios.

$\mathrm{O}$ não cumprimento das normas epidemiológicas na área gera um grande problema, podendo ressaltar algumas situações que contribui para a proliferação do protozoário da doença, como a ocorrência de terrenos baldios, a falta de destino adequado aos lixos causando entulhos pela cidade, falta de vigilância sanitária, o número de animais domésticos abandonados sem nenhum cuidado pela cidade são fatores contribuintes de proliferação já que os maiores casos de contaminação são através dos animais.

Quanto às ações de controle a serem tomadas para impedir a proliferação pode-se citar as medidas de controle e organização da saúde e zoonose, com capacitações específicas sobre o caso, gerando bons profissionais capacitados para auxiliar e orientar a população na prevenção e mitigação dos impactos gerados pela doença em humanos e animais, além do controle do vetor. Não deixando de lado a questão dos animais abandonados, promover ações políticas da região, que garantem a criação de casas de apoio (ONGs) a esses animais, com profissionais, alimentação e medicamentos, quando viável, podendo então receber os tratamentos necessários até que sejam adotados.

\section{Referências}

Almeida, C. P., Cavalcante, F. R. A., Moreno, J. D. O., Florêncio, C. M. G. D., Cavalcante, K. K. D. S., \& Alencar, C. H. (2020). Leishmaniose visceral: distribuição temporal e espacial em Fortaleza, Ceará, 2007-2017. Epidemiologia e Serviços de Saúde, 29.

Azevedo, R., de Silva, R. E., Costa, J. D. O. J., Pesenato, I. P., Souza, S. P. J., Castelli, G. S. N., ... \& Marcili, A. (2021). Leishmaniose Visceral no Brasil: o que é preciso saber. Brazilian Journal of Global Health, 3(1), 24-31.

Bastos, T., Madrid, D. M., \& Linhares, G. (2015). Aspectos gerais da leishmaniose visceral. Enciclopédia Biosfera, 11(22).

Benchimol, J. L., Gualandi, F. D. C., Barreto, D. C. D. S., \& Pinheiro, L. D. A. (2019). Leishmanioses: sua configuração histórica no Brasil com ênfase na doença visceral nos anos 1930 a 1960. Boletim do Museu Paraense Emílio Goeldi. Ciências Humanas, 14, 611-626.

Brasil. (2017). Ministério da Saúde. Secretaria de Vigilância em Saúde. Coordenação-Geral de Desenvolvimento da Epidemiologia em Serviços. Guia de Vigilância em Saúde. 2 ed. Brasília: Ministério da Saúde. 704 p.

Brasil. (2014). Ministério da Saúde. Secretaria de Vigilância em Saúde, Departamento de Vigilância Epidemiológica. Manual de vigilância e controle da leishmaniose visceral. Brasília: Editora do Ministério da Saúde, p. 120. 
Brasil. (2006). Ministério da Saúde. Secretaria de Vigilância em Saúde. Departamento de Vigilância Epidemiológica. Manual de vigilância e controle da leishmaniose visceral / Ministério da Saúde, Secretaria de Vigilância em Saúde, Departamento de Vigilância Epidemiológica. - Brasília: Editora do Ministério da Saúde.

Bueno, W. D. C. (2001). A cobertura de saúde na mídia brasileira: os sintomas de uma doença anunciada. Comunicação \& Sociedade, 22(35), 187-210.

Castro, A. G. D., Silva, A. R. D., Costa, C. H. N., \& Scherlock, I. (1996). Controle, diagnóstico e tratamento da leishmaniose visceral (calazar): normas técnicas. In Controle, diagnóstico e tratamento da leishmaniose visceral (Calazar): normas técnicas, pp. 86-86.

Costa, C. H. N., Pereira, H. F., \& Araújo, M. V. (1990). Epidemia de leishmaniose visceral no Estado do Piauí, Brasil, 1980-1986. Revista de Saúde Pública, $24,361-372$.

Lima, C. A. de, Teixeira, K. R., Moreira, J. P. F. F., \& Teixeira, K. R. (2013). Diagnóstico da leishmaniose visceral canina: uma revisão. PUBVET, 7, 25652677.

Dotta, S. C. N., Lot, R. F. E., \& Zappa, V. (2009). Métodos de diagnóstico da leishmaniose visceral canina. Revista Científica Eletrônica De Medicina Veterinária, (12).

Freitas, J. C. C. D., Nunes-Pinheiro, D. C. S., Lopes Neto, B. E., Santos, G. J. L., Abreu, C. R. A. D., Braga, R. R., ... \& Oliveira, L. F. D. (2012). Clinical and laboratory alterations in dogs naturally infected by Leishmania chagasi. Revista da Sociedade Brasileira de Medicina Tropical, 45, 24-29.

Genaro, O., Da Costa, C. A., Williams, P., Silva, J. E., Rocha, N. M., Lima, S. L., \& Mayrink, W. (1990). Ocorrência de calazar em área urbana da grande Belo Horizonte, MG. Revista da Sociedade Brasileira de Medicina Tropical, 23(2), 121-121.

Godoy, K. C. S., Braz, P. H., Assis, A. R., Antunes, T. R., Gomes, D. C., \& Souza, A. I. (2016). Avaliação dos indicadores de lesão miocárdica em cães com leishmaniose visceral. Arquivo Brasileiro de Medicina Veterinária e Zootecnia, 68, 313-320.

Gonçalves, A. J. R., Rozembaum, R., Cunha, R. Q. D., Menezes, J. A. D., Joäo Filho, E. C., Vieira, A. R. M., \& Carvalho, F. G. D. (1986). Calazar: relato de três pacientes adultos internados no HSE-INAMPS (RJ); consideraçöes sobre esta endemia de grande importância em nosso território. Arq. bras. med, 369-76.

Ibge (2010). Instituto Brasileiro de Geografia e Estatística. Disponível em: https://www.ibge.gov.br/cidades-e-estados/to/guarai.html.

Killick-Kendrick, R. (1990). Phlebotomine vectors of the leishmaniases: a review. Medical and veterinary entomology, 4(1), 1-24.

Leite, G. (1958). Leichimaniose visceral: I parte: proêmio, histórico e epidemiologia. Rev Bras Med, 15, 665-610

López, Y., Osorio, L., Alvarez, G., Rojas, J., Jiménez, F., Gómez, C., \& Ferro, C. (1996). Sandfly Lutzomyia longipalpis in a cutaneous leishmaniasis focus in central Colombia. Memórias do Instituto Oswaldo Cruz, 91, 415-419.

Malaquias, L. C. C., do Carmo Romualdo, R., do Anjos, J. B., Giunchetti, R. C., Corrêa-Oliveira, R., \& Reis, A. B. (2007). Serological screening confirms the re-emergence of canine leishmaniosis in urban and rural areas in Governador Valadares, Vale do Rio Doce, Minas Gerais, Brazil. Parasitology research, $100(2), 233-239$.

Mazucato, T. (2018). Metodologia da pesquisa e do trabalho científico. Penápolis: FUNEPE.

Morrison, A. C., Ferro, C., Pardo, R., Torres, M., Devlin, B., Wilson, M. L., \& Tesh, R. B. (1995). Seasonal abundance of Lutzomyia longipalpis (Dipteral Psychodidae) at an endemic focus of visceral leishmaniasis in Colombia. Journal of medical entomology, 32(4), 538-548.

Oliveira, H. (1960). Epidemiologia do Calazar. Revista brasileira de Medicina, 17(1), pp. 56-58.

Organização Pan-Americana da Saúde - OPAS. Leishmanioses: Informe Epidemiológico das Américas Nº Fevereiro 2018. Recuperado de <http://iris.paho.org/xmlui/bitstream/handle/123456789/34857/LeishReport6_por.pdf?seque5>.

Ortiz, R. C., \& Anversa, L. (2015). Epidemiologia da leishmaniose visceral em Bauru, São Paulo, no período de 2004 a 2012: um estudo descritivo. Epidemiologia e Serviços de Saúde, 24, 97-104.

Pessôa, S.B. (1967). Parasitologia médica. 7. ed. Rio de Janeiro:Guanabara Koogan,. Cap.15, pp. 161-184.

Pinto, A. J. W., Ribeiro, V. M., \& Tafuri, W. L. (2016). Análise do diagnóstico da leishmaniose visceral canina no Brasil, com ênfase no uso dos métodos sorológicos: teste imunocromatográfico, ELISA e reação de imunofluorescência indireta revisão de literatura. Clín. Vet., 80-86.

Rey, L.O. (1991). Parasitologia: parasitos e doenças parasitárias do homem.: O complexo "Leishmania donovani" e a leishmaniose visceral. 2. ed. Rio de Janeiro: Guanabara Koogan, cap.19.

Reis, L. L. D., Balieiro, A. A. D. S., Fonseca, F. R., \& Gonçalves, M. J. F. (2019). Leishmaniose visceral e sua relação com fatores climáticos e ambientais no Estado do Tocantins, Brasil, 2007 a 2014. Cadernos de Saúde Pública, 35.

Teixeira, K. K. T., do Nascimento, K. G., Santana, R. L., de Souza, A. M. G., de Souza, T. A., \& Barbosa, I. R. (2019). Padrões espaciais da ocorrência de leishmaniose visceral humana na cidade de Natal-RN: a influência das áreas de risco social. Hygeia-Revista Brasileira De Geografia Médica E Da Saúde, 15(32), 121- Uchôa 133.

Tesh, R. B. (1995). Control of zoonotic visceral leishmaniasis: is it time to change strategies?. The American journal of tropical medicine and hygiene, 52(3), 287-292.

World Health Organization - WHO. Disponível em: https://www.who.int/leishmaniasis/research/en/. 